\title{
Discusión entre disponibilidad energética y sustentabilidad ambiental en \\ Estados Unidos, 1980-2016
}

Discussion between energy availability and environmental sustainability in the United States, 1980-2016

\begin{tabular}{|l|l|l|}
\cline { 2 - 2 } & Alicia Puyana \\
$\begin{array}{l}\text { Facultad Latinoamericana } \\
\text { de Ciencias Sociales, } \\
\text { FLACSO }\end{array}$ & $\begin{array}{l}\text { Isabel } \\
\text { Rodríguez Peña } \\
\text { Universidad Anáhuac, } \\
\text { México }\end{array}$ \\
\hline
\end{tabular}




\section{Resumen}

La discusión sobre la seguridad energética (SE) se ha centrado en el mercado petrolero, sin embargo, la inclusión de temas ambientales ha derivado en un análisis complejo sobre las estrategias a implementar para garantizar la SE, en especial, para los países de alto consumo de fósiles y pocas o nulas reservas de petróleo, como Estados Unidos. El documento propone tres índices - petrolero, diversificación y ambiental-para evaluar la SE de Estados Unidos entre 1980 y 2016. Se concluye que, de acuerdo con la visión actual de la SE, solo podrán lograrse mejoras en esta si hay un incremento en energías renovables, ya que el aumento en el consumo de fósiles (especialmente de gas) no permiten transitar a una visión holística de SE.

Palabras clave: seguridad energética; índice de seguridad energética; energías renovables; diversificación energética.

Clasificación JEL: F52, 013, P48, L72.

\section{Abstract}

The core of the discussion for Energy Security (ES) has been the oil market, however, the inclusion of environmental issues has led to a complex analysis of the strategies to be implemented to guarantee ES, especially for countries with high consumption of fossils and few or not oil reserves like the United States. The document proposes three indexes - oil, diversification and environmental - to evaluate the US SE between 1980 and 2016. The document concludes that according to the current SE vision, improvements in the SE can only be obtained if there is an increase in renewable energies, because the increase in the consumption of fossil fuels (especially gas) does not allow a transition to a holistic SE vision.

Keywords: energy security; energy security index; renewable energy; energy diversification. JEL Classification: F52, 013, P48, L72. 


\section{Introducción}

Como muchas ideas y políticas relacionadas con las actividades económicas, políticas y sociales, la concepción, definición y desarrollo de seguridad energética (SE en adelante) ha adquirido complejidad, pari passu con la emergencia de nuevos problemas imposibles de ignorar, como el calentamiento global. A pesar de ello, desde inicios de los años setenta y hasta la fecha, los hidrocarburos se encuentran en el centro de la discusión en SE, de su gradual metamorfosis, así como de las políticas para garantizar los objetivos (International Energy Agency, IEA, 2019). Gran parte de ello se explica por la relevancia del petróleo en la matriz energética de cualquier nación, independientemente de su desarrollo.

Por la relevancia del petróleo en el consumo energético, el shock petrolero ocasionado por la Organización de Países Exportadores de Petróleo (OPEP) en la década de los setenta consolidó la relevancia del mercado petrolero en la discusión de SE. Sin embargo, la relación del consumo energético con las emisiones de $\mathrm{CO}_{2}$ ha derivado en la incorporación de temas relacionados con el medio ambiente y el calentamiento global. Lo anterior se tradujo en una visión holística de la SE, pero mucho más compleja.

Por esta razón, se ha dificultado la medición de la SE y la formulación de una guía para la implementación de políticas para asegurarla. Lo que se observa es que el centro de la discusión continúa siendo el mercado petrolero y la participación de fuentes fósiles, que se mantienen como los objetivos originales de la SE y relegan la incorporación de la sustentabilidad ambiental.

De acuerdo con lo planteado, en este artículo se incorpora literatura que se centra en formular una medida para evaluar la evolución de la SE. Entre ellos el MOSES (o Model of Short-Term Energy Security), publicado por la IEA (2011a); el índice de seguridad energética (Sovacool y Mukherjee, 2011); trilema energético, publicado por el World Energy Council (2007); el índex of U.S. Energy Security Risk, publicado por el Global Energy Institute (2019), entre otros. Las tres últimas metodologías tratan de dar ordenamiento a la relación entre asegurar las fuentes de energía con los objetivos ambientales, sin embargo, los resultados muestran que no se puede mejorar y garantizar la oferta energética sin incrementar las emisiones de $\mathrm{CO}_{2}$, a menos de que las garantías en la oferta energética provengan de energías renovables. 
En este contexto, el objetivo del documento es evaluar la SE en Estados Unidos en el período 1980-2016, a partir de la formulación de una propuesta compuesta por tres índices: índice de seguridad petrolera, índice de diversificación energética e índice de seguridad ambiental (ver Puyana y Rodríguez, 2019). La formulación de los tres índices está basada en una vasta revisión de la literatura, de la que se rescatan las principales discusiones de la evolución del concepto, especialmente, la importancia que ha mantenido el mercado petrolero y las contradicciones que surgen al incorporar la sustentabilidad ambiental en la evolución de la SE. De esta forma, nuestro planteamiento sigue de cerca la metodología del trilema energético y la diferencia respecto a este es que no formulamos un índice global, por lo que interpretamos el efecto que tiene cada uno de los índices en la SE. Además, para abordar la compleja discusión entre consumo de energía y medio ambiente, sin ser contradictoria, incorporamos en el índice de seguridad ambiental el consumo de fósiles como un determinante central en la reducción de $\mathrm{CO}_{2}$. De esta forma, queremos argumentar que de acuerdo con los debates actuales de $\mathrm{SE}$, las mejoras en este indicador no pueden estar únicamente basadas en una mayor disponibilidad de energías fósiles, ya que esto puede oprimir los avances en mejoras ambientales (los cuales se pueden relacionar con un incremento en las emisiones de $\mathrm{CO}_{2}$ ).

De la formulación de los tres índices se desprenden dos conclusiones. En primer lugar, la relevancia del mercado petrolero en la $\mathrm{SE}$, a pesar de la inclusión de nuevos temas (Puyana y Rodríguez, 2019). En segundo, las mejoras en la SE para Estados Unidos han sido resultado de una mayor producción y consumo de energías fósiles, lo cual deriva en un conflicto al considerar la parte ambiental. En este sentido, se muestra que la compleja relación entre disponibilidad de energía a precios aceptables y los efectos de un consumo mayor de energía en el medio ambiente es algo que puede abordarse en el mismo planteamiento, siempre y cuando la garantía de energía no se explique únicamente por el incremento de energía fósil.

Después de esta breve introducción, la investigación se desarrolla en tres secciones. En la siguiente se presenta el surgimiento de las discusiones de SE en el contexto del shock petrolero en la década de los setenta y la evolución e inclusión de los temas ambientales en la actualidad. Adicionalmente, a partir del reporte de US Energy Security Risk, se presenta la visión y objetivos implementados por Estados Unidos para asegurar la SE. En la sección tres se examina la propuesta y los resultados de la evaluación de la SE entre 1980 y 2016 en Estados Unidos. Por último, en la última sección se reúnen los principales hallazgos de la investigación. 


\section{La discusión de SE en la década de los setenta para el principal consumidor de energía}

Desde principios del siglo XX hasta inicios de la década de los setenta, el control de las reservas de petróleo estuvo en manos de las Siete Hermanas ${ }^{1}$ (Sampson, 1975, y Yergin, 1991). Esto no se consideró un problema ni económico ni político, ya que dominaban toda la cadena del petróleo, exploración, producción, refinación y la distribución al consumidor final. Solo en 1973, la posesión y distribución geográfica de los yacimientos de hidrocarburos conocidos pasó a ser una amenaza para la seguridad nacional de las potencias económicas, que concentraban el $40 \%$ del consumo mundial. La declaratoria de la OPEP en 1973 del embargo petrolero llevaba a restringir exportaciones mediante un sistema de cuotas que afectaba la distribución de crudo entre las principales potencias económicas, aquellas que eran las grandes importadoras de crudo.

Por la elevada intensidad petrolera del PIB, la elasticidad precio de la demanda de petróleo era baja, casi nula, y el fuerte incremento en los precios elevó la inflación en los países importadores, especialmente los más industrializados y los mayores consumidores, como era el caso de Estados Unidos. El embargo elevó los precios del petróleo de 2.7 a 10.4 dólares el barril, ocasionando una masiva transferencia de ingresos de los países importadores hacia los exportadores. La gráfica 1 ejemplifica el efecto económico del shock petrolero en las economías con mayor consumo de petróleo, entre ellos, Estados Unidos, Francia y el Reino Unido entre 1960 y 1990 (ver gráfica 1).

1 El cartel de las siete hermanas estaba compuesto por las siguientes empresas petroleras: Standard Oil of New Jersey (después Exxon), Royal Dutch Shell, the Anglo-Persian Oil Company (APOC, después BP), Standard Oil of New York (Socony, después Mobil), Standard Oil of California (Socal, después Chevron), Gulf Oil y Texaco. 
Gráfica 1. Tasa anual de crecimiento de los precios al consumidor en Estados Unidos, Francia y el Reino Unido (serie desestacionalizada, eje izquierdo, y precios del petróleo en dólares por barril, eje derecho, 1960 y 1990)

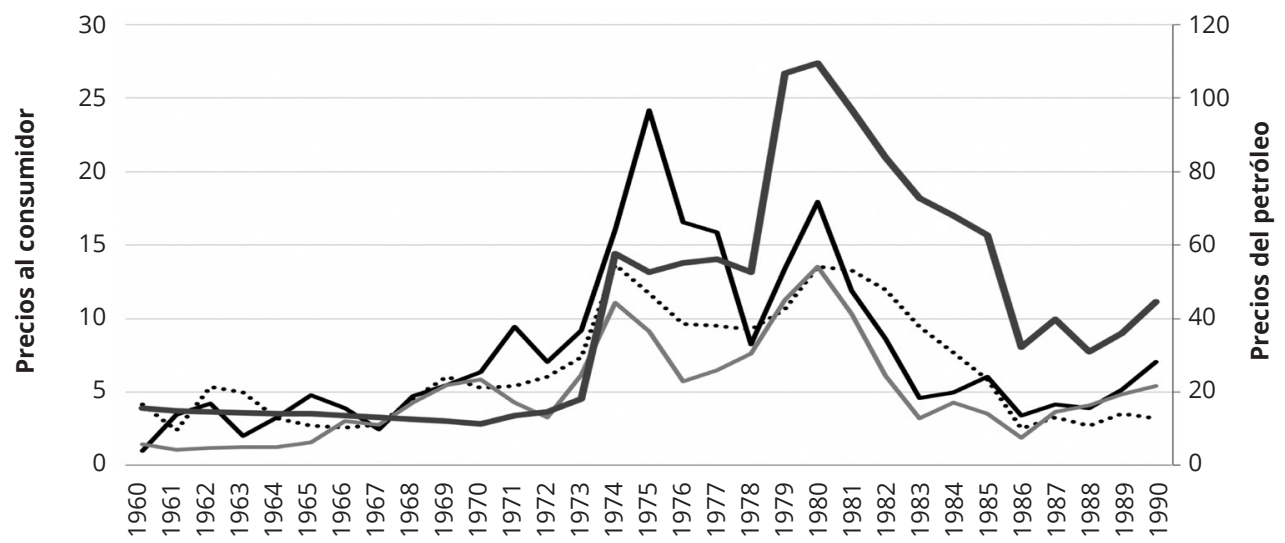

R. Unido ……... Francia — E. Unidos - Precios del petróleo

Fuente: elaboración propia con datos de la Federal Reserve Bank of St. Louis (2018) y BP (2019).

Varios factores explican la consolidación de la estrategia de la OPEP de revalorar el petróleo y establecer su poder de mercado mediante un recorte en la oferta. Por este lado de la oferta, en primer lugar, debido a la alta concentración de las reservas ubicadas, principalmente en países del Oriente Medio, que representaban el 64 \% de las reservas mundiales en la década de los setenta; en segundo lugar, la notable diferencia de costos de exploración, desarrollo y producción de la OPEP, en especial de los países del golfo Pérsico, Irán, Irak y Libia, así como los costos de las actividades aguas arriba (exploración, desarrollo de campos y extracción) de crudo de la mayoría de los países de la OPEP, eran y son considerablemente menores a los del resto del mundo (Puyana, 2015). Por el lado de la demanda, la elevada dependencia petrolera de la economía mundial debido a que el petróleo permeó toda la estructura económica global, el transporte y las fuerzas armadas.

En este contexto, surge la discusión y se convierte en una parte de la seguridad nacional. La primera formalización de SE, en acuerdos internacionales adoptados con relativa transparencia, se dio en noviembre de 1974, con la creación de IEA, suscrita por 15 países industrializados. Estados Unidos, como principal consumidor de petróleo y energía en general, fue el más activo en el debate sobre las medidas a tomar a fin de contrarrestar los efectos de los cambios en el mercado 
energético mundial a raíz de la política de la OPEP en 1973 (National Security Council, 1979). En situación similar se encontraban Europa y Japón, cuya mayor dependencia de las importaciones de crudo los orilló a sellar alianzas con Estados Unidos y coordinar sus políticas ante la estrategia de la OPEP.

La IEA define la SE como el acceso ininterrumpido a las fuentes de energías a un precio promedio. Sus objetivos se han centrado en evitar interrupciones no planeadas del suministro de petróleo que desequilibren las economías, al inducir efectos en la cadena productiva de bienes y servicios, lo que podría amenazar la seguridad interna (IEA, 2019). Los mecanismos que ha planteado han sido, en primer lugar, establecer un mercado de libre competencia en la energía; desarrollo de sistemas de información y programas comunes -entre miembros y no miembros-; diversificación de fuentes de energía y mejoras en la eficiencia de energía. ${ }^{2}$

En Estados Unidos el debate derivó en la creación de la Energy Information Administration (EIA) en 1977. Sus objetivos se plantearon con el fin de mantener y mejorar los sistemas para enfrentar las interrupciones en el suministro de petróleo; mejorar el sistema de oferta y demanda de energía mediante el desarrollo de fuentes alternativas y la promoción de la eficiencia en el uso de petróleo. En el plano internacional se concentraron en promover la cooperación, en este ámbito, en tecnologías energéticas y en la integración de las políticas energéticas entre los países miembros. Todas estas medidas se impusieron con el fin de reducir los riesgos impuestos por la OPEP.

Por lo tanto, para garantizar la SE, los miembros de la OCDE, representados por la IEA y, específicamente, Estados Unidos con la EIA, se han comprometido en políticas de SE con acciones y metas precisas, siempre en el marco de un mercado energético libre. Los compromisos comprenden acciones de corto y largo plazo de los gobiernos para mitigar los riesgos por falta de disponibilidad física o debido al alza

\footnotetext{
2 Los riesgos que afectan la SE de los países miembros y de la comunidad internacional provienen de: 1) Las restricciones en la capacidad productiva y de refinación. Se considera que aun un recorte pequeño en el suministro de crudo o de productos refinados ocasiona alzas de precios en toda la economía. 2) Para la organización, el resurgimiento del nacionalismo y la consecuente politización de las políticas de los mayores productores es un riesgo, ya que limita el libre ejercicio del mercado. 3) Tensiones geopolíticas y terrorismo, las cuales añaden tensiones e inestabilidad al mercado. Para la EIA, la región más crítica es el Medio Oriente, en donde han ocurrido las crisis de mayor impacto (Krane y Medlock, 2018).
} 
de precios por escalada de la demanda. En el corto plazo, las medidas incluyen el establecimiento de reservas estratégicas y planes de contingencia para reducir el consumo cuando ocurra interrupción en el suministro. Por otro lado, las políticas tienden a centrarse en abordar las causas profundas de la inseguridad energética relacionadas con la concentración de las reservas y la producción en unos pocos países, algunos de los cuales son considerados políticamente inestables o no amistosos. Tales medidas van desde ampliar la producción propia de crudo, fomentar las fuentes alternativas y el abaratamiento de los costos de exploración, desarrollo y producción que hacen viables campos menos rentables.

\section{Discusión contemporánea de SE; incorporación de temas medioambientales}

En un inicio la discusión se centró en medidas para prevenir el agotamiento de las reservas de petróleo establecidas, principalmente, por Estados Unidos, en la década de los setenta. A principios del presente siglo, la discusión se complejizó al incorporar temas medioambientales, surgidos en encuentros como el IPCC (Intergovernmental Panel on Climate Change) y la Conferencia de París. Específicamente, en 2015, el acceso a la energía se consideró un derecho humano en sí mismo... al vincular la energía asequible con la garantía de otros derechos humanos básicos: «el derecho a la vida, la alimentación, la vivienda, la salud, la educación, etc. Todos estos están tupidamente entrelazados con el acceso a servicios eficientes de energía» (Pandey, 2018, traducción propia). Así lo ratifican los 17 Objetivos de Desarrollo Sostenible, todos los cuales dependen del objetivo 7: «Garantizar el acceso a energía asequible, segura, sostenible y moderna para todos» (Naciones Unidas, 2015).

En este sentido, la literatura contemporánea sobre SE es más amplia y compleja en comparación con la discusión de la década de los setenta y con la propuesta de la IEA plasmada en el MOSES. ${ }^{3}$ En la actualidad, se incluyen los riesgos vinculados

\footnotetext{
3 EI MOSES es una herramienta integral para medir la SE. Se centra en el análisis de los riesgos y la resiliencia asociados a las interrupciones físicas a corto plazo del suministro de energía (IEA, 2011a). Incluye en su análisis la composición de la oferta energética en términos de riesgos externos e internos y la resiliencia externa e interna. Entre los principales factores dentro de esas clasificaciones están la dependencia de la importación neta y la estabilidad política de los proveedores y, respecto de la resiliencia, considera la cantidad de puntos de entrada para un país (por ejemplo, puertos y oleoductos), el nivel de inventarios y la diversidad de proveedores. Además, se consideran
} 
a la naturaleza (escasez de recursos y eventos naturales extremos); técnica (envejecimiento de infraestructura, accidentes tecnológicos); políticos (restricción intencional de suministros o tecnologías, sabotaje y terrorismo), y económico (por ejemplo, la reciente volatilidad de los precios del petróleo). Entre las propuestas que intentan capturar las preocupaciones actuales y proponer un análisis holístico de la SE se encuentran: el World Energy Trilemma Index, ${ }^{4}$ publicado por el World Energy Council desde 2007 y, recientemente, los trabajos de Sovacool y Mukherjee (2011), Sovacool (2013) y Winzer (2012) plantean la propuesta del índice de SE. 5 Una de las características de ambos planteamientos es la inclusión de las preocupaciones medioambientales como parte del análisis energético.

A pesar de la incorporación de temas ambientales y de gobernanza en los análisis actuales de $\mathrm{SE}$, se observa que la discusión aún se centra en lo que fue su detonante en la década de los setenta: el mercado petrolero. Y es que, a pesar de las medidas puestas en marcha y de los logros obtenidos, la predominancia del petróleo en la estructura energética y económica continúa en el centro de la discusión sobre cómo garantizar la SE. ${ }^{6}$ Así lo ratifica el reporte de Energy Security Risk, publicado por el Global Energy Institute de Estados Unidos.

los principales mecanismos que deberían implementar las instituciones internacionales para hacer frente a eventuales interrupciones en el suministro de petróleo mediante un sistema de información permanente en el mercado internacional del petróleo y sugiere la promoción de otras energías dentro de la oferta energética.

${ }^{4}$ Esta metodología, cuyos orígenes se remontan a los primeros informes del IPCC, proponen un índice de SE compuesto por tres categorías: equidad energética (accesibilidad y asequibilidad del suministro de energía en toda la población), equidad energética (accesibilidad y asequibilidad; eficiencia de la energía por el lado de la oferta y la demanda, desarrollo de energías renovables y otras fuentes de energías bajas en carbono) y sostenibilidad ambiental (eficiencia de la energía por el lado de la oferta y la demanda, desarrollo de energías renovables y otras fuentes de energías bajas en carbono).

${ }^{5}$ Consiste en la recopilación de una amplia y compleja lista de 320 indicadores subdividida en cinco clasificaciones: disponibilidad, asequibilidad, desarrollo de tecnología y eficiencia, ambiente y sostenibilidad, y regulación y gobernanza.

6 En general los combustibles fósiles han concentrado la demanda de energía y su proporción osciló en torno al 93 \% entre 1940 y 1973. A partir de entonces se contrajo paulatinamente hasta el $80 \%$ en 2017, es decir, 13 puntos porcentuales en 45 años. Se espera que para 2040 descienda a 69 \%. La caída de los combustibles fósiles se explica, en primer lugar, por el descenso del petróleo, el cual ha pasado de concentrar más de $45 \%$ del consumo en la década de los setentas, período 
Las preocupaciones derivadas del shock petrolero y las políticas que ha implementado Estados Unidos para hacer frente a dicho cambio de régimen se pueden reflejar en el U.S. Energy Security Risk, reporte que provee una vasta base de datos (compuesta por 37 variables) para evaluar la SE de 1970 a la actualidad en Estados Unidos. Sus resultados se expresan en cuatro subíndices: geopolítico, económico, fiabilidad y ambiental, los cuales a su vez componen el índice global de SE. Los subíndices se consideran áreas en las cuales debe centrarse la política energética para garantizar la seguridad nacional.

El Energy Security Risk, metodología confiable para medir la SE de Estados Unidos, tiene como propósito identificar los indicadores y factores que podrían mejorar o empeorar la situación de ese país, con el objetivo de lograr su independencia energética. Considera 1980 como año base, debido a que es considerado el de mayor riesgo energético, especialmente por la reducida cantidad de reservas de petróleo. Dado este proceso de normalización, a medida que los índices se acercan al número 100, el riesgo energético se incrementa. ${ }^{7}$

en el cual se registra el mayor nivel, a una reducción de 37 en 2017. Cabe señalar que, durante los 45 años considerados, la reducción no ha sido ni constante ni lineal, lo cual indica que el consumo petrolero responde a diversos factores, en buena medida a la dinámica de la economía y a la de los precios del petróleo, la evolución de la tecnología y factores políticos diversos (EIA, 2019). Respecto dell resto de la oferta de fósiles, el carbón ha descendido a una tasa no mayor a 1.5 \%, a pesar de las presiones por descarbonizar las economías (en especial, la reducción de carbón por tratarse de la energía más contaminante). El gas muestra una tendencia creciente a nivel mundial, y gran parte se debe a que las reservas se encuentran mejor distribuidas entre los países y debido a que el gran consumidor de energía, Estados Unidos, ha devenido en uno de los principales productores de gas. En suma, la ligera reducción del petróleo y el carbón se ha visto contrarrestada por la producción de gas, lo que se traduce en una magra reducción de los combustibles fósiles a pesar de las preocupaciones que causó el shock petrolero y de las recientes preocupaciones por el cambio climático (ver gráfica 1 del anexo).

7 Debido a la forma en la que se normaliza el índice no queda acotado entre 0 y 100 . Por ello, en algunos años el índice queda por arriba del 100, lo cual indica que la situación del indicador es peor que en el año 1980. 
Gráfica 2. Evolución de los subíndices y el índice de riesgo energético, 1980-2022

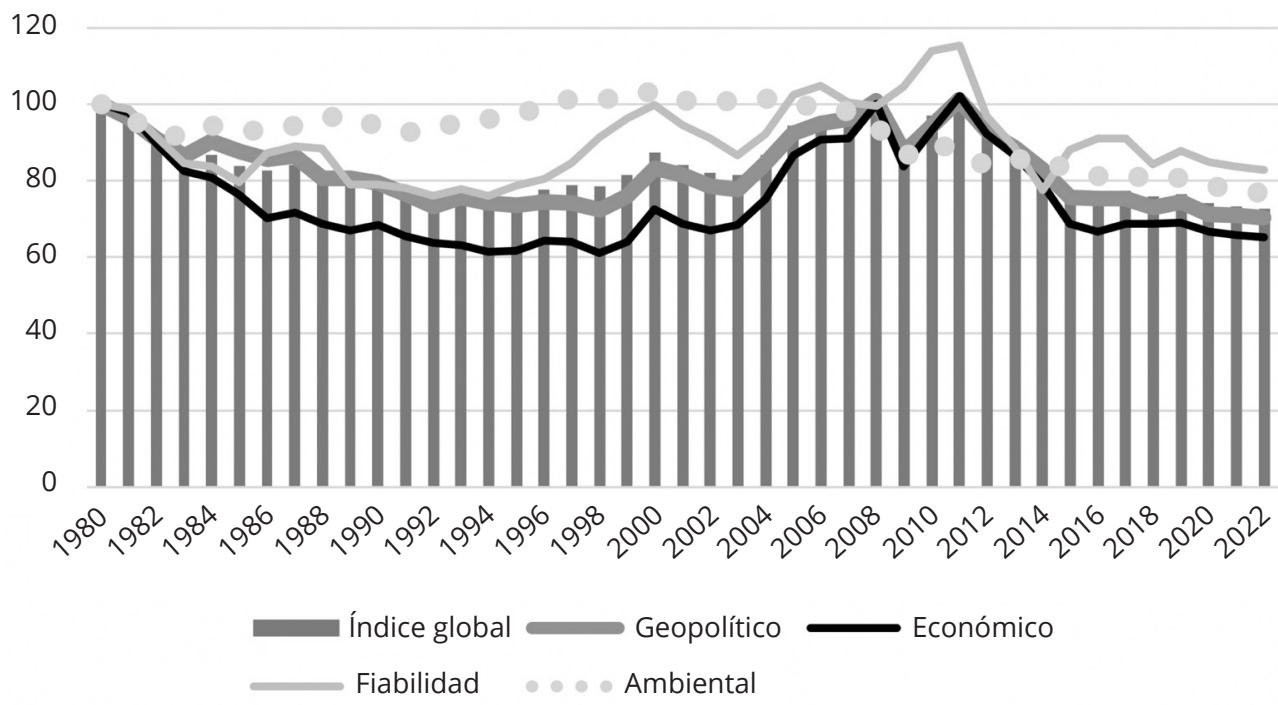

Fuente: Global Energy Institute (2019).

De acuerdo con el informe del 2019, las perspectivas, en comparación con las experiencias de alto riesgo energético en la década de los setenta y principio de los ochenta, han mejorado considerablemente; así lo refleja la evolución de los cuatro subíndices y el índice global (véase la gráfica 2). El subíndice geopolítico se construye a partir, entre otras variables, de las reservas de petróleo, 8 lo cual muestra la visión inicial de SE de la EIA. ${ }^{9}$ Por lo tanto, las mejoras en el índice global y, especialmente, en el geopolítico, se deben al cambio tecnológico en procesos de exploración que se ha reflejado en la agregación de reservas y producción de fósiles. Además, la

8 El índice geopolítico se construye a partir de las principales fuentes energéticas (petróleo, gas y carbón). Estas tres fuentes toman variables como reservas, producción, importaciones, precios, entre otros.

9 Debido a su condición como importador neto de petróleo y gas y, como se planteó en la sección pasada, al impacto del shock petrolero en los setenta, no es de extrañar la relevancia que tiene el tema geopolítico en el índice. Para Estados Unidos, la SE siempre ha estado planteada a partir de la preocupación por lograr independencia en la importación de petróleo, en especial de aquellos países que considera políticamente inestables. 
ponderación de los subíndices geopolítico y estratégico (de $30 \%$, respectivamente) tienen una fuerte influencia en los resultados globales. Por ello, la tendencia de ambos es muy similar.

Es importante notar que, a pesar del incremento en la producción de petróleo continua siendo un importador neto, por ello la inestabilidad del precio del petróleo continúa afectando la SE en Estados Unidos.

En lo que respecta a la parte ambiental, la cual solo aporta $20 \%$ en el índice global de $\mathrm{SE}$, a inicios del presente siglo se observó un incremento considerable de las energías alternativas, que representan el $10 \%$ (porcentaje que incluye fuentes hidroeléctricas) del total de energía primaria. Por otra parte, el incremento continuo de energía fósil, a pesar de los avances en eficiencia energética, se refleja en la evolución ascendente del $\mathrm{CO}_{2}$. Esto último puede asociarse al incremento en la demanda del sector trasporte.

Por lo tanto, a pesar de los intentos por incorporar los temas ambientales. Se observa que la metodología del Energy Security Risk prioriza la cantidad de reservas, producción y consumo de fósiles como factores centrales en la evolución de la SE. Siguiendo esa lógica, se concluye que hay avances importantes en la SE, especialmente en comparación con la década de los setenta y principios de los ochenta, sin embargo, si se trata de abordar la SE a partir de las discusiones actuales, los resultados son ambiguos. Por ello creemos que el argumento de Cherp y Jewell (2012), en el Global Energy Assessment, es central para entender la complejidad de la SE en la actualidad, ya que muchos de los países persiguen rigurosamente las agendas de SE sin estrategias sólidas en temas de cambio climático, a pesar de que ambos estén relacionados. Los efectos son bidireccionales, por ejemplo, el cambio climático afecta la disponibilidad de agua para la generación de energía hidroeléctrica y térmica, o la inestabilidad marítima ocasionada por ciclones provoca el cierre de las plataformas. A pesar de la complejidad del tema, las preocupaciones por el cambio climático pueden ser un detonante en el fomento de energías alternativas, estrategia que, indirectamente, terminará por reducir la dependencia de los combustibles fósiles y la promoción de fuentes de energía renovables. A continuación se presenta el análisis que proponemos para evaluar la SE en Estados Unidos. 


\section{Definición de índices para analizar la evolución de la SE en Estados Unidos entre 1980-2016}

Esta sección se basa en el documento de Puyana y Rodríguez (2019), en el cual se plantean las bases para la formulación de los índices de seguridad petrolera (ISP), diversidad energética (IDE) y seguridad ambiental (ISA). ${ }^{10}$ Estos se implementaron para analizar la evolución de la SE en Estados Unidos entre 1980 y 2016. En la sección anterior se mostró que el detonante de la discusión de la SE en la década de los setenta fue el shock petrolero. A pesar de los esfuerzos por diversificar y lograr mayor eficiencia, la evolución y pronósticos de la composición de la matriz energética muestran que el actor central continuará siendo el petróleo. Por ello, el mercado petrolero expresado en el ISP será central en el análisis que proponemos para analizar la evolución de la SE.

La construcción del ISP se plantea a partir de tres variables compuestas. a) La primera es el consumo aparente entre el PIB (producto interno bruto). A su vez, el consumo aparente es igual al consumo más las importaciones, menos exportaciones de petróleo y petrolíferos, dividido entre el PIB real, base 2010. Las variables se transformaron en millones de dólares con el objetivo de relacionarlas con el PIB. Una vez construida la variable compuesta, se le asocia a un valor negativo en la participación del índice. b) Vida útil de las reservas, igual al cociente de reservas probadas de petróleo entre la producción anual, expresado en millones de barriles anuales. Se considera el valor positivo. c) Dependencia de las importaciones, que se formula a partir del cociente de importaciones de petróleo y petrolífero entre el consumo aparente (variable que se construyó en el inciso a), en millones de dólares. Se considera el valor negativo.

De acuerdo con la formulación del ISP, las mejoras en la SE están relacionadas con una menor dependencia de las importaciones de petróleo y petrolíferos, una menor intensidad petrolera (una reducción en el consumo de petróleo por unidad de PIB) e incrementos en las reservas mejoran el índice. Estados Unidos, como el principal consumidor de energía, considera centrales estas variables en su política energética de las últimas décadas.

10 En el documento de Puyana y Rodríguez (2019), el ISA se plantea como ITE, sin embargo, su construcción es la misma. 
Otro elemento importante para mejorar la SE ha sido la diversificación de las fuentes de energía. Por ello, se propone el Índice de Diversidad Energética (IDE), que representa la diversidad de fuentes de energía e incluye: petróleo, gas, carbón y energías renovables. Del cálculo se excluye la nuclear, debido a que no ha registrado cambios relevantes en las últimas décadas y no es clara su evolución futura. Mediante este indicador se trata de abordar el tema de diversificación como una estrategia para mejorar la SE. En el caso del petróleo y el gas, se incluyen las mismas que en el cálculo del ISP; en la evolución del carbón se considera la producción menos consumo en mtoe (millones de toneladas de petróleo equivalente) y para medir las energías renovables solo se incluye el consumo anual en mtoe. En este caso, una mayor IDE se relaciona con mayores reservas, menores importaciones de petróleo, gas y carbón y, por último, mayor consumo.

Debido a la relación entre consumo de energía y emisiones de $\mathrm{CO}_{2}$ es que se construye el Índice de Seguridad Ambiental (ISA). Su lógica es que las mejoras del indicador dependerán, básicamente, de dos variables: la reducción en el consumo de fósiles y el mayor uso de energías alternativas. De esta forma se establece que una proporción importante en las emisiones de $\mathrm{CO}_{2}$ está asociada con el consumo de energía fósil. Se construye a partir de tres variables: la emisión de $\mathrm{CO}_{2}$, el consumo de fósiles y la producción de energías alternativas en mtoe. Las dos primeras se incorporan en el cálculo con símbolo negativo, pues señala su relación con el consumo de fósiles y, en consecuencia, los efectos que tiene con la producción de $\mathrm{CO}_{2}$. La última, con símbolo positivo, debido a que representa una transición energética baja en emisiones de $\mathrm{CO}_{2}$.

En la figura 1 se desglosan las variables compuestas y simples que se incluyen en cada uno de los índices. Se formularon seis variables compuestas para el caso del petróleo y el gas, que a su vez incluyen seis variables simples. En total, la formulación de los tres índices suma 18 variables simples.

El mecanismo para normalizar las variables que se incluyen en los tres índices consiste en el cociente de cada una de las variables en las que el denominador será el valor más grande en el período. Una vez normalizadas las series, se le asigna un valor negativo o positivo, dependiendo de su relación con el índice. Por ejemplo, en el ISA, a las emisiones de $\mathrm{CO}_{2}$ y al incremento en el consumo en fósiles se les asigna un valor negativo, debido a que afectan negativamente la evolución del indicador. Con esta metodología, los resultados que se obtienen se encuentran acotados entre cero y uno, y la lectura es que, a medida que se encuentre más cerca del 1 hay mejoras en el ISP, IDE e ISA. En el anexo, se resumen los valores obtenidos de acuerdo con la normalización. El sombreado en cada una de las celdas muestra el valor mayor y menor en el período de estudio. 
The Anáhuac Journal: Business and Economics, Vol. 20, núm. 1, 2020.

https://doi.org/10.36105/theanahuacjour.2020v20n1.02

Figura 1. Variables que componen los índices para evaluar la SE

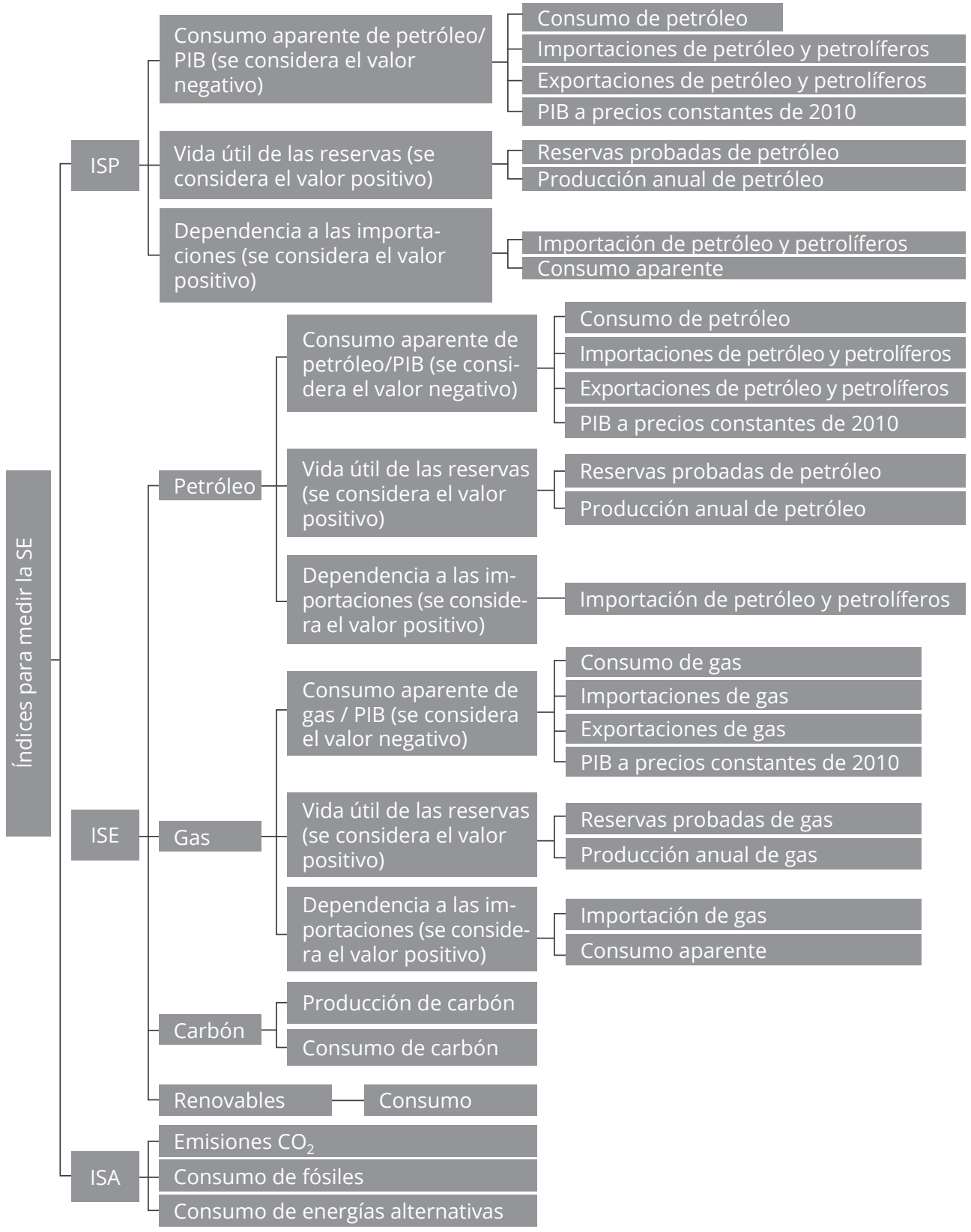


En términos metodológicos, estamos de acuerdo con Kim (2015) respecto al concepto abstracto de $\mathrm{SE}$, el cual podría entenderse con facilidad, sin embargo, hay una dificultad apremiante al medirlo. Por ello, con el objetivo de tener un resultado robusto, la información que se incluye en los índices que proponemos son variables numéricas del mercado energético y excluimos temas de geoestretegia, política o gobernanza. Con ello no queremos decir que estos temas no sean relevantes para analizar la evolución de la SE, por el contrario, reconocemos su importancia y los incorporamos en la lectura de los índices.

Por último, la propuesta plantea un índice de SE basado en diferentes bloques de variables, los cuales no se mezclan para definir un índice general. Esto marca una diferencia con los planteamientos del trilema energético y, especialmente, con el Energy Security Risk. Este último plantea la formulación de un índice global y, además, pondera la participación de los subíndices en el global de acuerdo con la relevancia en la discusión central de SE (el geopolítico y económico representan 30 \%, respectivamente, y $20 \%$ el ambiental). ${ }^{11}$

\section{La evolución de la SE en Estados Unidos entre 1980 y 2016}

El shock petrolero impulsó a Estados Unidos a mejorar la eficiencia en el consumo de petróleo, lo cual se materializó en distintas estrategias para lograr, en primer lugar, diversificar las fuentes $y$, en segundo, diversificar los oferentes de petróleo. En especial, las estrategias se centraron en reducir la dependencia hacia aquellos países que atentaban contra su seguridad nacional. Con las estrategias se intentó a toda costa transformar la estructura productiva y mejorar la eficiencia energética, sin embargo, a pesar de las distintas estrategias impuestas, este país continúa siendo el mayor consumidor de crudo del mundo, con 20.5 millones de barriles diarios que corresponden a $21 \%$ del consumo mundial (BP, 2019).

La evolución del consumo no siempre fue lineal. En gran parte su dinámica ha dependido del crecimiento económico, y así se mostró durante la crisis financiera de

11 Estas ponderaciones no han cambiado durante todo el período en el que se presentan los resultados del índice 1970. Las ponderaciones dan un peso sustancial a cada uno de los cuatro subíndices, pero otorgan un peso mayor a los riesgos geopolíticos y económicos, los cuales han dominado el debate sobre SE. 
2008. Sin embargo, en la actualidad continúa siendo uno de los principales consumidores de energía primaria, solo después de China. En lo que respecta al consumo de derivados del petróleo y gas, lidera la lista, y en carbón descendió del segundo al tercer lugar durante el período de estudio. A partir del presente siglo, Estados Unidos se ha consolidado como uno de los principales productores de energía renovable, sin embargo, este avance no ha permitido que se ubique entre los primeros diez en generación de energía eléctrica mediante fuentes alternativas a las fósiles (Enerdata, 2019). Los pronósticos muestran que esta tendencia se modificará hacia 2040, y que las energías renovables, sumadas a la generación hidroeléctrica, superarán en 30 \% la energía para Estados Unidos (ver gráfica 3).

Gráfica 3. Consumo de energía primaria en Estados Unidos, 1970-2016

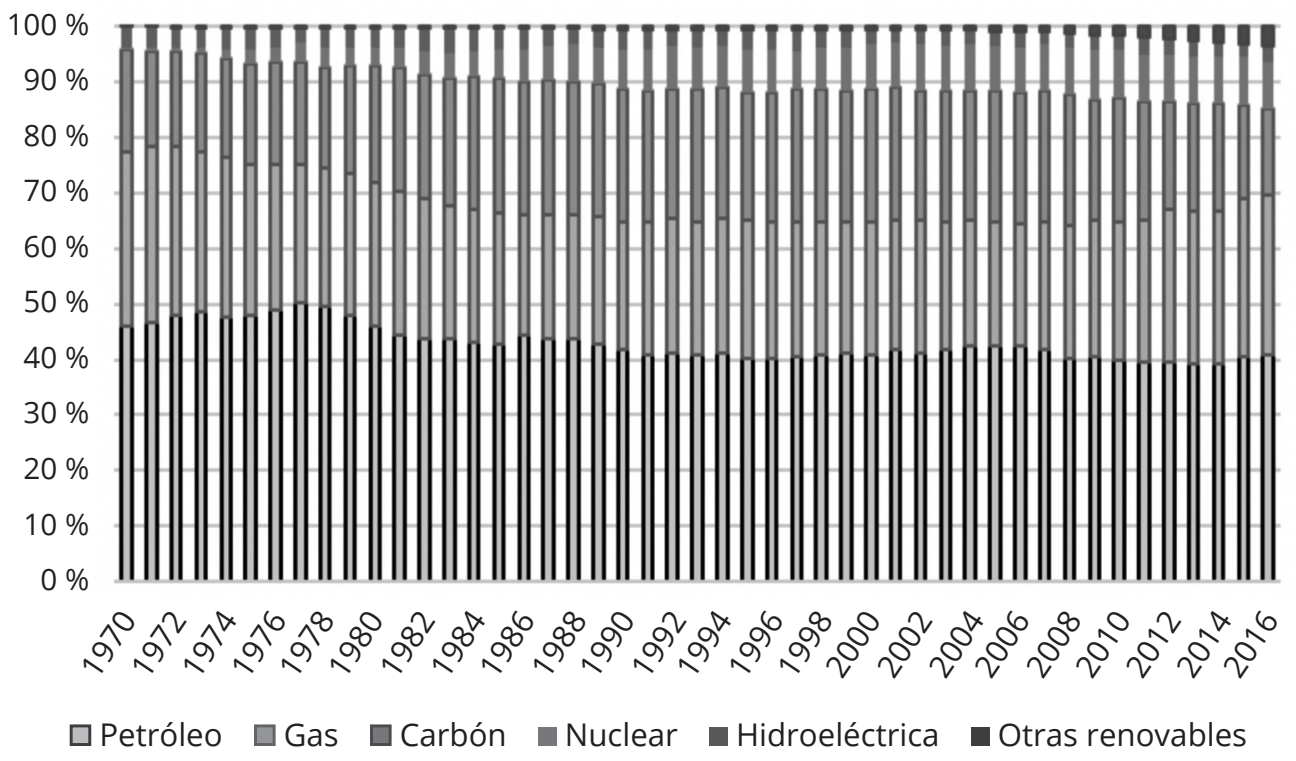

Fuente: elaboración propia con información de Energy Information Administration, 2019.

Los datos anteriores muestran que, a pesar de las políticas impuestas con el shock petrolero en los años setenta, enfocadas básicamente en reducir la dependencia petrolera, las estrategias para garantizar la SE estuvieron centradas en mantener el consumo de fósiles y solo ahora se observa una mayor relevancia de las energías renovables. 
Lo que cambió radicalmente a partir del shock petrolero fue su posición como oferente de energía. De acuerdo con el Global Energy Statistical Yearbook (Enerdata, 2019), es el principal productor de crudo a pesar de que sus reservas se encuentran entre 2 y $2.5 \%$ del total mundial durante el período de estudio.

El actor principal en lo que se ha considerado una nueva era para Estados Unidos en términos energéticos es el gas, porque el fracking lo consolidó como el principal productor desde 2013, además de que este producto ganó posición en el del consumo energético. Respecto a la producción de carbón, el país se ha mantenido como el segundo productor a nivel mundial, a pesar de tratarse de la energía más contaminante.

Los resultados, de acuerdo con la composición de los índices, se analizan a continuación. En la gráfica 4 se muestran los resultados para el ISE, el cual se ha mantenido en un rango considerablemente bueno entre .5 y .9. Se observa una recuperación del índice entre 1986 y 2008, que se asocia a mejoras en la intensidad energética (expresado en el consumo aparente/PIB), a mejoras en el saldo comercial de productos derivados del petróleo y de petróleo crudo, además de al ligero incremento en las reservas de petróleo. ${ }^{12}$ Se advierten caídas importantes en 1986, 2008 y 2014. En 1986 se registra el peor índice durante el período de estudio y este se explica por el descenso de 3 \% en la producción de petróleo; por su parte, 2008 se asocia a una caída importante en la dinámica en la economía que afectó la intensidad energética y una caída en las reservas probadas de $-11 \%$ en 2014 . En general se perciben mejoras en el índice debido a una menor dependencia de petróleo y sus derivados, y a mejoras en la intensidad energética, ambos conceptos centrales para las mejoras en SE (ver gráfica 4).

\footnotetext{
12 Entre 1980 e inicios del presente siglo, la tasa de crecimiento de las reservas fue continua, pero a una tasa negativa (de -1.2 \% entre 1980 y 1990 y -2 \% entre 1990 y 2000). De 2000 a la actualidad la tasa ha sido de $2 \%$.
} 
Gráfica 4. Índice de seguridad petrolera, 1980-2016

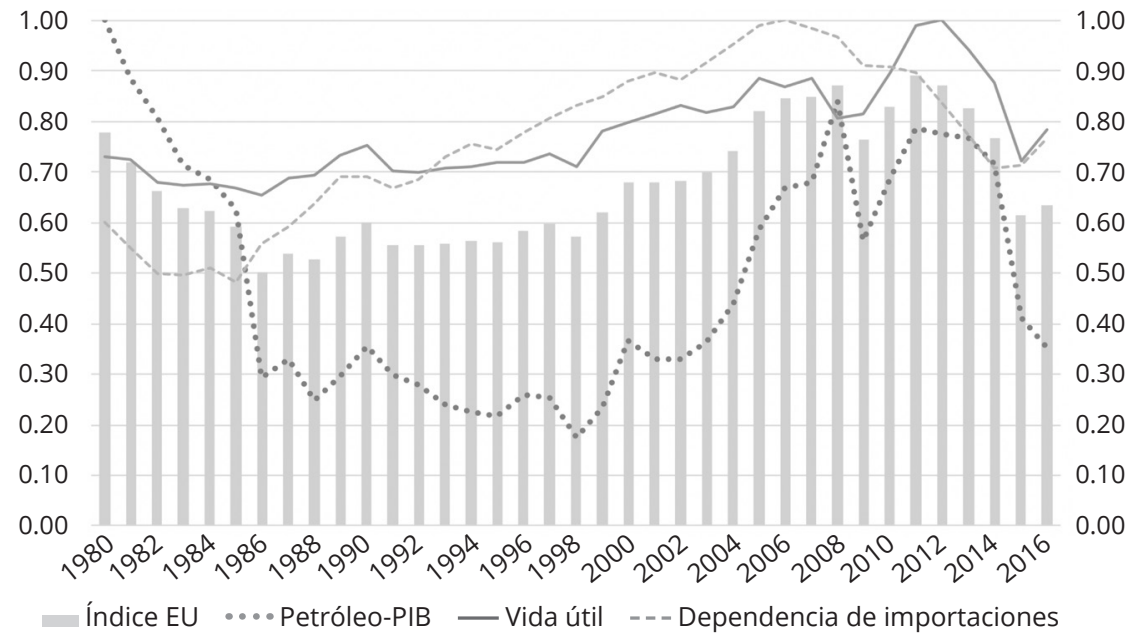

Fuente: elaboración propia con datos de BP (2019).

El IDE oscila entre 3 y .7 (ver gráfica 5). La relativa estabilidad del índice se explica por el incremento en la producción y consumo de petróleo, gas y carbón, lo que para Estados Unidos ha sido central en su política energética. La estabilidad de este indicador se asocia con un concepto más general y, especialmente, con las discusiones en la década de los setenta, en el que una mayor diversificación de fuentes fósiles implica un mayor consumo de y acceso a energía, y se traduce en mejoras en la SE.

Gráfica 5. Índice de diversidad energética, 1980 - 2016

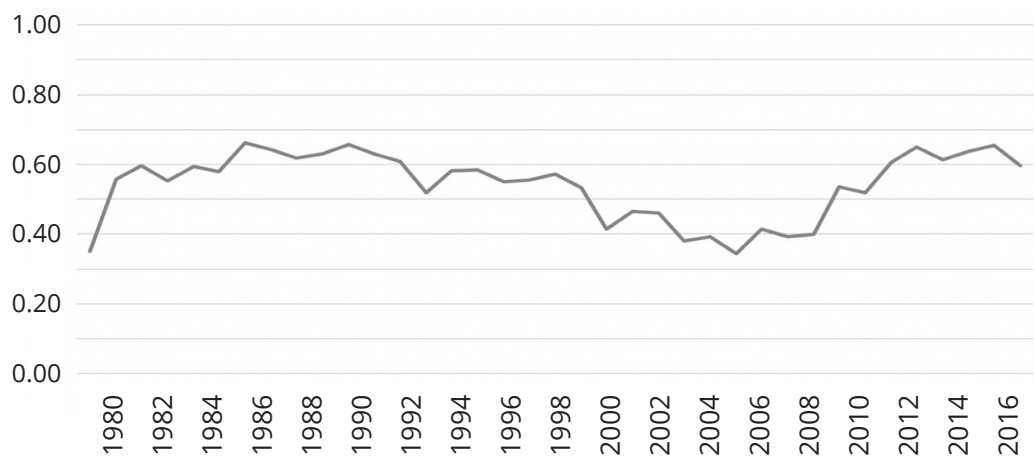

Fuente: elaboración propia con información de BP (2019). 
En lo que concierne a la diversificación, es de gran relevancia el incremento en la producción de gas y crudo de lutitas desde inicios del presente siglo, bonanza productiva que ubicó a Estados Unidos como el primer productor gas en el mundo. ${ }^{13}$ Durante el siglo pasado, la explotación del gas se consideraba económicamente inviable, sin embargo, a inicios de 2007 las principales petroleras estadounidenses ExxonMobil, Chevron y Halliburton, y otras grandes industrias en el mundo, empezaron a invertir cientos de millones de dólares en propaganda que justifica y defiende el fracking, a pesar de los daños que este tiene sobre el medio ambiente (Bacchetta, 2013). Se espera que la producción no convencional se extienda en los siguientes años y, de acuerdo con las estimaciones de la EIA, la producción de Shale ${ }^{14}$ estará concentrada en seis países, Estados Unidos el principal. De acuerdo con el Global Energy Institute (2019) la combinación de fractura hidráulica y perforación horizontal en un contexto de incremento en las reservas del gas de lutitas, anunciaban que estaba por lograrse la independencia energética de Estados Unidos. Lo anterior se refleja en mejoras considerables en la SE y en un importante impacto en lo económico y geopolítico. En este contexto, el gas ha marcado un avance crucial en la SE de Estados Unidos, por una parte, por el incremento considerable de reservas y producción de la segunda energía más consumida para el país y, por otra, al lograr independencia, alcanza mejoras considerables en el tema de la resiliencia y la capacidad de responder ante desequilibrios del mercado petrolero.

13 La capacidad energética que ha alcanzado Estados Unidos en las últimas décadas ha alentado a algunos autores a denominarlo la revolución energética del país con el mayor consumo de energía (Kobek, Ugarte y Aguilar, 2015).

14 Derivado del gas. 
Gráfica 6. Producción menos consumo de gas y petróleo, 1980-2016

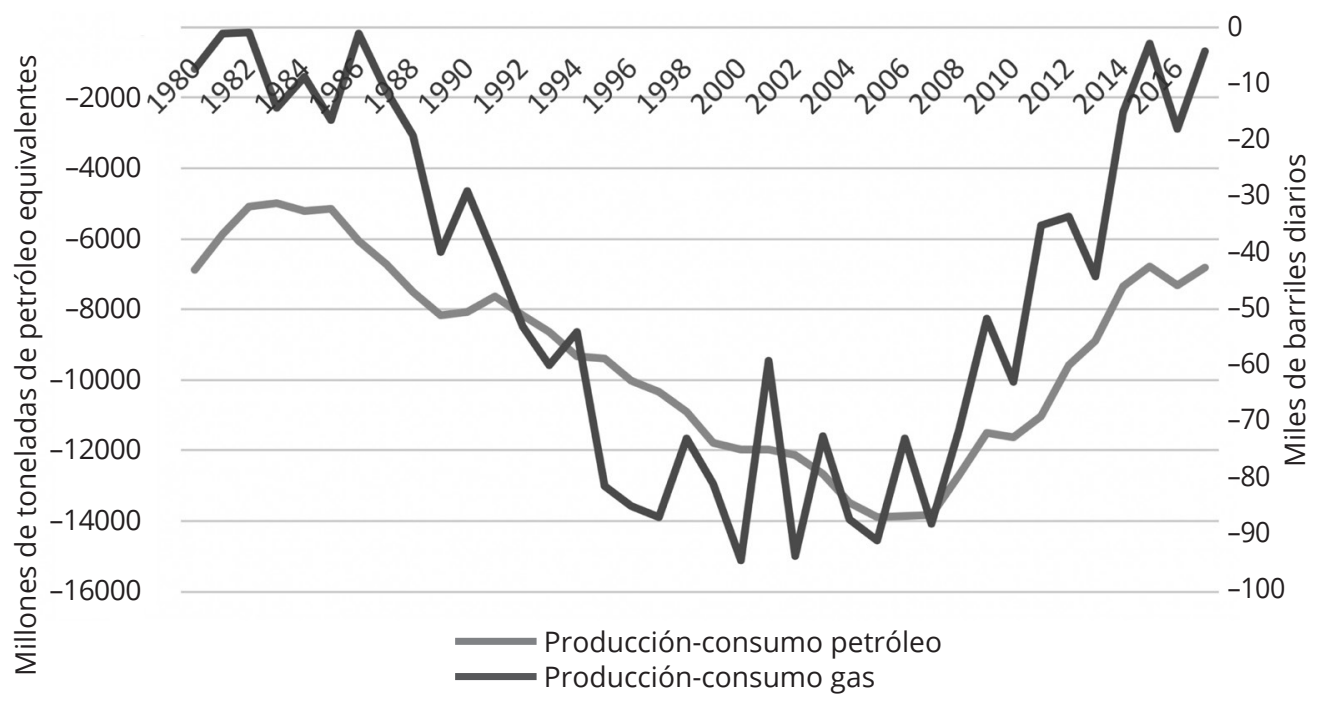

Fuente: elaboración propia con datos de la BP (2019).

Sin embargo, continúa siendo un importador neto de petróleo (ver gráfica 6) y, en consecuencia, los cambios de los precios del crudo afectan su economía, a pesar de las ganancias en eficiencia y diversificación. Por ello, la inestabilidad del Medio Oriente (como el referente geopolítico en la determinación del precio de las principales mezclas de petróleo en el mundo) continúa siendo central para la estabilidad de su sector energético y por seguridad nacional. Así también lo refleja el reporte Energy Security Risk. Existen otros dos efectos del precio del petróleo sobre el mercado energético. Por un lado, la dinámica entre el precio de este y la evolución de las energías renovables. Cuando los precios del petróleo son muy bajos, desestimulan la entrada de fuentes más costosas, fósiles (por ejemplo, el petróleo proveniente de Estados Unidos o del mar del Norte y otros productores no convencionales de petróleo) y alternativas (debido a que, en cuanto a costos, siempre será más eficiente optar por los fósiles), desincentivando la diversificación. Cuando los precios suben, son un aliciente para la diversificación, sin embargo, la proporción que tienen en la matriz energética ocasiona presiones en la SE. ${ }^{15}$ Por otro, la mayor inestabilidad

15 Se ha mostrado que una reducción en los precios del petróleo deriva en un desincentivo de las energías alternativas, en especial, aquellas cuyos costos superan los de las energías fósiles 
del precio del petróleo ocasionada por la financiarización de las materias primas ${ }^{16}$ tiene efectos similares a los antes descritos. En términos generales, la inestabilidad del precio del petróleo puede tener efectos indirectos sobre las emisiones de $\mathrm{CO}_{2}$; repercusiones en el fomento de energías renovables; desequilibrios externos en economías dependientes de las importaciones que tiendan a agravarse causando inflación y estancamiento económico, entre otros.

Por último, el ISA refleja el incremento en la producción de energías renovables. Estados Unidos se encuentra entre los tres principales productores de energías renovables en el mundo, sin embargo, el consumo nacional de estas no supera el $6 \%$ del total mundial, mientras tanto los fósiles continúan dominando la matriz energética. Lo anterior resulta en el sostenido incremento de emisiones de gases de efecto invernadero y de $\mathrm{CO}_{2}$ asociados al consumo de fósiles. De acuerdo con el Global Carbon Atlas (2019), Estados Unidos fue el principal emisor de $\mathrm{CO}_{2}$ hasta 2005 (con más de seis millones de toneladas, lo cual supera en casi dos toneladas lo que emiten los 28 países de la Unión Europea). Actualmente, solo se encuentra por debajo de China (que emite diez millones de toneladas de $\mathrm{CO}_{2}$ ) y ambos países contribuyen en $43.8 \%$ a nivel mundial. Lo anterior se refleja en la gráfica 7, con un ascenso paulatino de las emisiones de $\mathrm{CO}_{2}$ durante el período de estudio, mostrando un valor máximo en el primer quinquenio del presente siglo.

Por el lado del componente que aporta positivamente al ISA, se observa el incremento considerable en energías renovables a partir de 2000, las cuales, para 1980, aún no figuraban en la composición de la matriz energética (principalmente la solar, eólica y biocombustibles, ya que la energía hidráulica en la década de los setenta representa cerca de $40 \%$ y, actualmente, solo $20 \%$ ). Las medidas y estrategias por diversificar las fuentes de energías renovables se materializaron prácticamente al empezar el presente siglo, en el que se muestra un incremento considerable de la energía eólica, la solar y los biocombustibles en los últimos 15 años. De alguna

-petróleo o gas- (EIA, 2018). Lo anterior refleja la importancia y el dominio que tiene el petróleo en la composición del complejo mercado energético, a pesar de la emergencia de las energías alternativas y el incremento en la producción del gas.

${ }^{16}$ A inicios de la crisis de 2008, la intromisión de los actores especulativos en los mercados de materias primas (ante la inestabilidad de los mercados de valores tradicionales), especialmente el petróleo, abrió paso a investigaciones centradas en la relación entre los mercados financieros y estas, que demuestran cómo la especulación guio el precio de las materias (Hamilton, 2009, y Cheng y Xiong, 2014). 
manera, estos avances se deben a los altos precios de las energías fósiles, los cuales mostraron valores récord en el período de estudio y se convirtieron en un aliciente para el fomento de energías alternativas. Esto podría significar un cambio importante para la SE, sin causar efectos colaterales en los temas ambientales, es decir, un resultado más congruente con las discusiones actuales de SE. Sin embargo, la evolución del ISA, por debajo de cero, muestra que falta mucho para que Estados Unidos, como gran productor y consumidor de fósiles y el segundo mayor emisor de $\mathrm{CO}_{2}$, logre resultados en conjunto (ver gráfica 7).

Gráfica 7. Determinantes del ISA e ISA

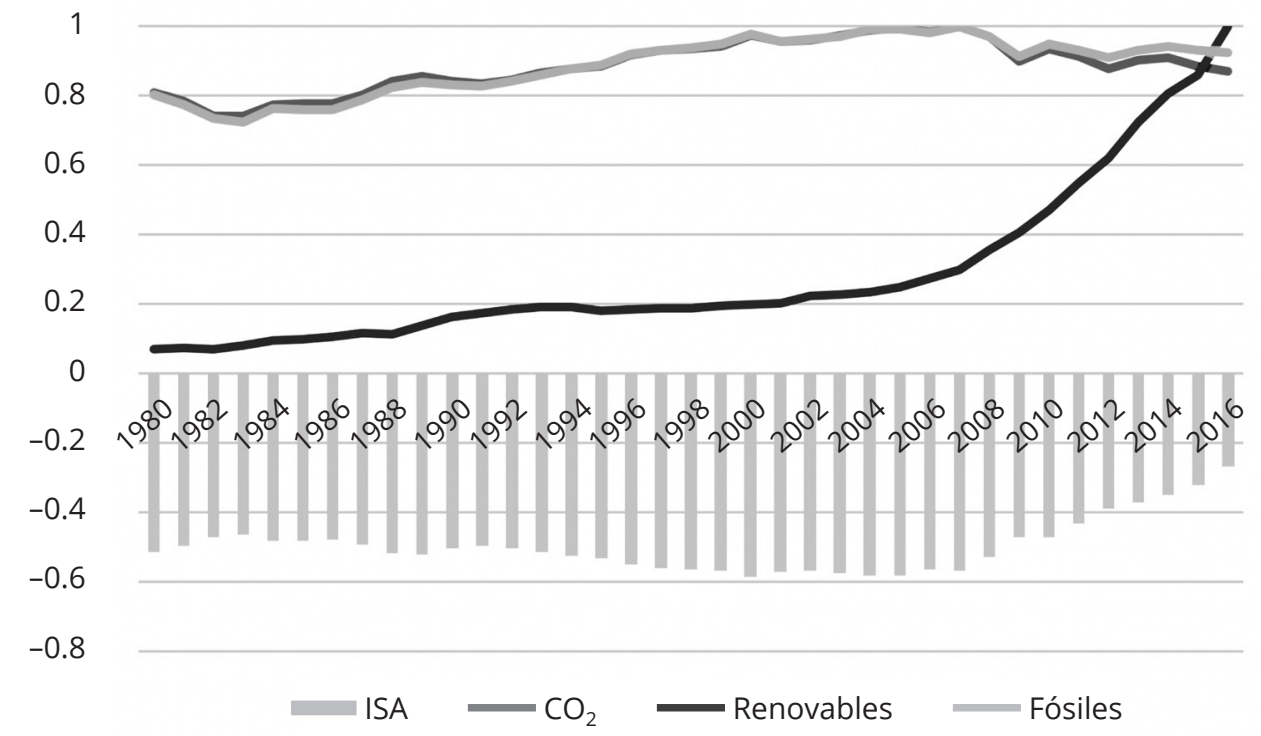

Fuente: elaboración propia.

En términos generales, respecto a la oferta energética, a más de 40 años del shock petrolero en 1973, Estados Unidos ha reducido sus importaciones de crudo y gas. Ahora es uno de los países que promueven la energía renovable, sin embargo, se estimula ante todo la industria petroquímica y el consumo de fósiles (ver gráfica 3), el cual se ha mantenido cercano al $90 \%$ del total de energía consumida. En este contexto, en comparación con la década de los setenta, Estados Unidos ha logrado incrementar su oferta energética. 
En el tema ambiental, la decisión de incrementar la producción de combustibles fósiles y anular muchas disposiciones ambientales, y de retirarse del Acuerdo de París sobre el Cambio Climático muestra la poca prioridad que confiere a esos temas. Estados Unidos ha mantenido una posición activa en la construcción de un modelo energético que garantice el acceso ininterrumpido a la energía. Esto se ha reflejado en un mayor incremento en la producción de gas natural, la extracción de crudo y mayor número de refinadoras, sin importar los problemas ambientales relacionados con el fracking (Baccheta, 2013). Sin embargo, no se observa un cambio constante en su política para darle prioridad al tema ambiental. Así lo corrobora el reporte Energy Security Risk. Otro factor que ha causado un incremento en la demanda de energía fósil a pesar de las mejoras en intensidad energética ha sido el mayor consumo de energía para el transporte, que actualmente representa cerca de $30 \%$ del consumo de energía, respecto al 20 \% en 1995 (Energy Information Agency, 2019).

\section{Conclusión}

Distintos documentos oficiales e investigaciones científicas han planteado que el detonante para las discusiones de SE en la década de los setenta fue el shock petrolero (1973) impuesto por la OPEP. A partir de ese suceso, los mecanismos para garantizar la SE han estado centrados en asegurar el acceso a la energía, especialmente al petróleo. Sin embargo, las preocupaciones por el cambio climático han derivado en una reformulación de la SE. Un planteamiento holístico y ambicioso que plantea correctamente la discusión entre energía y medio ambiente, pero continúa priorizado el consumo de energía fósil.

En este contexto, la presente investigación ha identificado que la realidad de la política energética en Estado Unidos dista de los objetivos internacionales que actualmente se han planteado para garantizar una visión holística de SE. Lo anterior se deduce de la formulación de los tres índices que proponemos que, mediante un ordenamiento distinto, incorpora la evolución de las variables ambientales en función del consumo de fósiles. Además, debido a la relevancia que tiene el mercado petrolero dentro de la matriz energética, se considera como variable central en la evolución de la SE.

Los resultados obtenidos en esta investigación son contrastantes con los del Energy Security Risk. Por una parte, coincidimos en los avances en la diversificación, y así lo ratifica la estabilidad del IDE. La formulación de este índice se asocia con un concepto 
más general y, especialmente, con las discusiones en la década de los años setenta, en los que una mayor diversificación de fuentes fósiles (en el caso de Estados Unidos, un incremento importante en las reservas y producción de gas, aunando a un incremento considerable en petroquímicos) implica un mayor consumo/acceso de energía, lo cual se traduce en mejoras en la SE pero a su vez presiona cualquier avance en términos ambientales, especialmente si los avances en SE son logrados a partir del consumo de fósiles.

Por lo tanto, el problema con la diversificación en un avance constante en la SE es que no se han priorizado las energías renovables; es decir, a pesar de su incremento en energías renovables, estas no representan un peso importante en el consumo global. De esta manera, se concluye que las nuevas discusiones de SE deberían dirigir la política en la materia hacia una transición que desincentive las energías fósiles que tanto frenan los avances en favor del medio ambiente. 


\section{Anexo}

Cuadro A1. Normalización de variables para la formalización del índice

\begin{tabular}{|c|c|c|c|c|c|c|c|c|c|c|c|}
\hline \multirow{2}{*}{ Años } & \multicolumn{3}{|c|}{ Petróleo } & \multicolumn{3}{|c|}{ Gas } & \multirow{2}{*}{$\begin{array}{c}\text { Carbón } \\
\begin{array}{c}\text { Consu- } \\
\text { mo }\end{array}\end{array}$} & \multirow{2}{*}{$\begin{array}{c}\text { Reno- } \\
\text { vables } \\
\text { Consu- } \\
\text { mo }\end{array}$} & \multirow{2}{*}{$\frac{\mathrm{CO}_{2}}{\text { Emisión }}$} & \multirow{2}{*}{$\begin{array}{c}\text { Reno } \\
\begin{array}{c}\text { Consu- } \\
\text { mo }\end{array}\end{array}$} & \multirow{2}{*}{$\begin{array}{c}\text { Fósiles } \\
\begin{array}{c}\text { Consu- } \\
\text { mo }\end{array} \\
\end{array}$} \\
\hline & $\begin{array}{l}\text { Petró- } \\
\text { leo-PIB }\end{array}$ & $\begin{array}{c}\text { Vida } \\
\text { útil }\end{array}$ & D_I & $\begin{array}{c}\text { GAS/ } \\
\text { PIB }\end{array}$ & $\begin{array}{c}\text { Vida } \\
\text { útil }\end{array}$ & D_I & & & & & \\
\hline & & & & & & & MToe & MToe & MToe & MToe & MToe \\
\hline 1980 & $9.34 \%$ & 9.5 & $23.4 \%$ & 0.0 & 10.3 & 0.0 & & 6.2 & 4773.5 & 6.0 & 1644.5 \\
\hline 1981 & $8.27 \%$ & 9.4 & $21.5 \%$ & 0.0 & 10.5 & 0.0 & 59.1 & 6.0 & 4611.3 & 6.2 & 1590.4 \\
\hline 1982 & $7.53 \%$ & 8.8 & $19.4 \%$ & 0.0 & 11.3 & 0.1 & 79.4 & 6.7 & 4374.6 & 6.0 & 1504.3 \\
\hline 1983 & $6.67 \%$ & 8.7 & $19.3 \%$ & 0.0 & 12.5 & 0.1 & 32.4 & 8.1 & 4358.7 & 6.7 & 1487.9 \\
\hline 1984 & $6.39 \%$ & 8.8 & $19.9 \%$ & 0.0 & 11.4 & 0.0 & 63.4 & 8.2 & 4557.8 & 8.1 & 1568.4 \\
\hline 1985 & $5.84 \%$ & 8.7 & $18.8 \%$ & 0.0 & 11.8 & 0.1 & 44.2 & 8.8 & 4573.7 & 8.2 & 1559.4 \\
\hline 1986 & $2.75 \%$ & 8.5 & $21.7 \%$ & 0.0 & 12.0 & 0.0 & 53.8 & 9.7 & 4585.5 & 8.8 & 1559.4 \\
\hline 1987 & $3.09 \%$ & 8.9 & $23.1 \%$ & 0.0 & 11.3 & 0.1 & 51.1 & 9.6 & 4728.6 & 9.7 & 1617.0 \\
\hline 1988 & $2.33 \%$ & 9.0 & $24.9 \%$ & 0.0 & 9.9 & 0.1 & 45.3 & 11.6 & 4948.8 & 9.6 & 1688.0 \\
\hline 1989 & $2.76 \%$ & 9.5 & $26.9 \%$ & 0.0 & 9.7 & 0.1 & 54.5 & 13.7 & 5037.9 & 11.6 & 1719.2 \\
\hline 1990 & $3.32 \%$ & 9.8 & $26.9 \%$ & 0.0 & 9.5 & 0.1 & 78.6 & 14.5 & 4953.0 & 13.7 & 1705.9 \\
\hline 1991 & $2.79 \%$ & 9.1 & $26.0 \%$ & 0.0 & 9.4 & 0.1 & 62.3 & 15.4 & 4907.6 & 14.5 & 1694.6 \\
\hline 1992 & $2.62 \%$ & 9.1 & $26.7 \%$ & 0.0 & 9.3 & 0.1 & 60.0 & 15.9 & 4986.8 & 15.4 & 1729.8 \\
\hline 1993 & $2.24 \%$ & 9.2 & $28.5 \%$ & 0.0 & 8.8 & 0.1 & 9.9 & 15.9 & 5097.3 & 15.9 & 1765.8 \\
\hline 1994 & $2.11 \%$ & 9.2 & $29.5 \%$ & 0.0 & 8.7 & 0.1 & 52.7 & 15.2 & 5174.7 & 15.9 & 1800.4 \\
\hline 1995 & $2.02 \%$ & 9.3 & $29.0 \%$ & 0.0 & 8.9 & 0.1 & 46.5 & 15.5 & 5205.4 & 15.2 & 1824.4 \\
\hline 1996 & $2.42 \%$ & 9.3 & $30.3 \%$ & 0.0 & 8.7 & 0.1 & 40.3 & 15.8 & 5386.9 & 15.5 & 1884.6 \\
\hline 1997 & $2.36 \%$ & 9.6 & $31.4 \%$ & 0.0 & 8.8 & 0.1 & 42.3 & 15.7 & 5474.3 & 15.8 & 1910.5 \\
\hline
\end{tabular}


The Anáhuac Journal: Business and Economics, Vol. 20, núm. 1, 2020.

https://doi.org/10.36105/theanahuacjour.2020v20n1.02

(continúa)

\begin{tabular}{|c|c|c|c|c|c|c|c|c|c|c|c|}
\hline \multirow{2}{*}{ Años } & \multicolumn{3}{|c|}{ Petróleo } & \multicolumn{3}{|c|}{ Gas } & \multirow{2}{*}{\begin{tabular}{|c|} 
Carbón \\
$\begin{array}{c}\text { Consu- } \\
\text { mo }\end{array}$ \\
\end{tabular}} & \multirow{2}{*}{$\begin{array}{c}\text { Reno- } \\
\text { vables } \\
\text { Consu- } \\
\text { mo }\end{array}$} & \multirow{2}{*}{$\frac{\mathrm{CO}_{2}}{\text { Emisión }}$} & \multirow{2}{*}{$\begin{array}{c}\text { Reno } \\
\begin{array}{c}\text { Consu- } \\
\text { mo }\end{array}\end{array}$} & \multirow{2}{*}{$\begin{array}{c}\text { Fósiles } \\
\begin{array}{c}\text { Consu- } \\
\text { mo }\end{array}\end{array}$} \\
\hline & $\begin{array}{l}\text { Petró- } \\
\text { leo-PIB }\end{array}$ & $\begin{array}{l}\text { Vida } \\
\text { útil }\end{array}$ & D_I & $\begin{array}{l}\text { GAS/ } \\
\text { PIB }\end{array}$ & $\begin{array}{c}\text { Vida } \\
\text { útil }\end{array}$ & D_I & & & & & \\
\hline & & & & & & & MToe & MToe & MToe & MToe & MToe \\
\hline 1998 & $1.64 \%$ & 9.2 & $32.4 \%$ & 0.0 & 8.8 & 0.1 & 54.6 & 16.2 & 5508.8 & 15.7 & 1921.7 \\
\hline 1999 & $2.20 \%$ & 10.1 & $33.1 \%$ & 0.0 & 8.9 & 0.2 & 37.4 & 16.5 & 5553.9 & 16.2 & 1947.5 \\
\hline 2000 & $3.41 \%$ & 10.4 & $34.2 \%$ & 0.0 & 9.2 & 0.2 & 1.0 & 16.8 & 5726.9 & 16.5 & 2001.9 \\
\hline 2001 & $3.07 \%$ & 10.6 & $34.9 \%$ & 0.0 & 9.4 & 0.2 & 36.2 & 18.7 & 5623.5 & 16.8 & 1960.9 \\
\hline 2002 & $3.09 \%$ & 10.8 & $34.4 \%$ & 0.0 & 9.9 & 0.2 & 17.2 & 18.8 & 5652.8 & 18.7 & 1978.8 \\
\hline 2003 & $3.39 \%$ & 10.6 & $35.7 \%$ & 0.0 & 9.9 & 0.2 & -8.4 & 19.7 & 5725.7 & 18.8 & 1989.6 \\
\hline 2004 & $4.11 \%$ & 10.8 & $37.2 \%$ & 0.0 & 10.4 & 0.2 & 5.9 & 20.7 & 5826.5 & 19.7 & 2031.2 \\
\hline 2005 & $5.48 \%$ & 11.5 & $38.6 \%$ & 0.0 & 11.3 & 0.2 & 5.4 & 22.8 & 5863.7 & 20.7 & 2034.1 \\
\hline 2006 & $6.25 \%$ & 11.3 & $38.7 \%$ & 0.0 & 11.4 & 0.2 & 28.0 & 24.8 & 5787.9 & 22.8 & 2010.8 \\
\hline 2007 & $6.34 \%$ & 11.5 & $38.4 \%$ & 0.0 & 12.3 & 0.2 & 13.7 & 29.7 & 5881.4 & 24.8 & 2048.9 \\
\hline 2008 & $7.86 \%$ & 10.5 & $37.7 \%$ & 0.0 & 12.1 & 0.2 & 30.9 & 33.9 & 5704.0 & 29.7 & 1989.4 \\
\hline 2009 & $5.27 \%$ & 10.6 & $35.5 \%$ & 0.0 & 13.2 & 0.2 & 42.3 & 39.3 & 5295.8 & 33.9 & 1873.7 \\
\hline 2010 & $6.42 \%$ & 11.6 & $35.3 \%$ & 0.0 & 14.2 & 0.2 & 24.9 & 45.7 & 5508.3 & 39.3 & 1945.9 \\
\hline 2011 & $7.36 \%$ & 12.9 & $34.9 \%$ & 0.0 & 14.5 & 0.1 & 57.7 & 51.7 & 5374.7 & 45.7 & 1911.3 \\
\hline 2012 & $7.25 \%$ & 12.9 & $32.7 \%$ & 0.0 & 12.8 & 0.1 & 75.9 & 60.2 & 5168.6 & 51.7 & 1864.1 \\
\hline 2013 & $7.16 \%$ & 12.2 & $30.0 \%$ & 0.0 & 13.9 & 0.1 & 44.0 & 67.2 & 5309.1 & 60.2 & 1912.6 \\
\hline 2014 & $6.69 \%$ & 11.4 & $27.6 \%$ & 0.0 & 14.2 & 0.1 & 51.5 & 71.5 & 5360.1 & 67.2 & 1931.3 \\
\hline 2015 & $3.85 \%$ & 9.4 & $27.8 \%$ & 0.0 & 11.3 & 0.1 & 54.6 & 83.1 & 5214.4 & 71.5 & 1909.9 \\
\hline 2016 & $3.30 \%$ & 10.2 & $29.8 \%$ & 0.0 & 12.1 & 0.1 & 7.8 & 94.8 & 5129.5 & 83.1 & 1893.3 \\
\hline
\end{tabular}

Fuente: elaboración propia con información de la BP (2019). 
Gráfica A 1. Demanda mundial de energía primaria 1949-2017. Estructura en porcentajes respecto al total de energía (eje izquierdo) y consumo de fósiles respecto al total de energía (eje derecho)
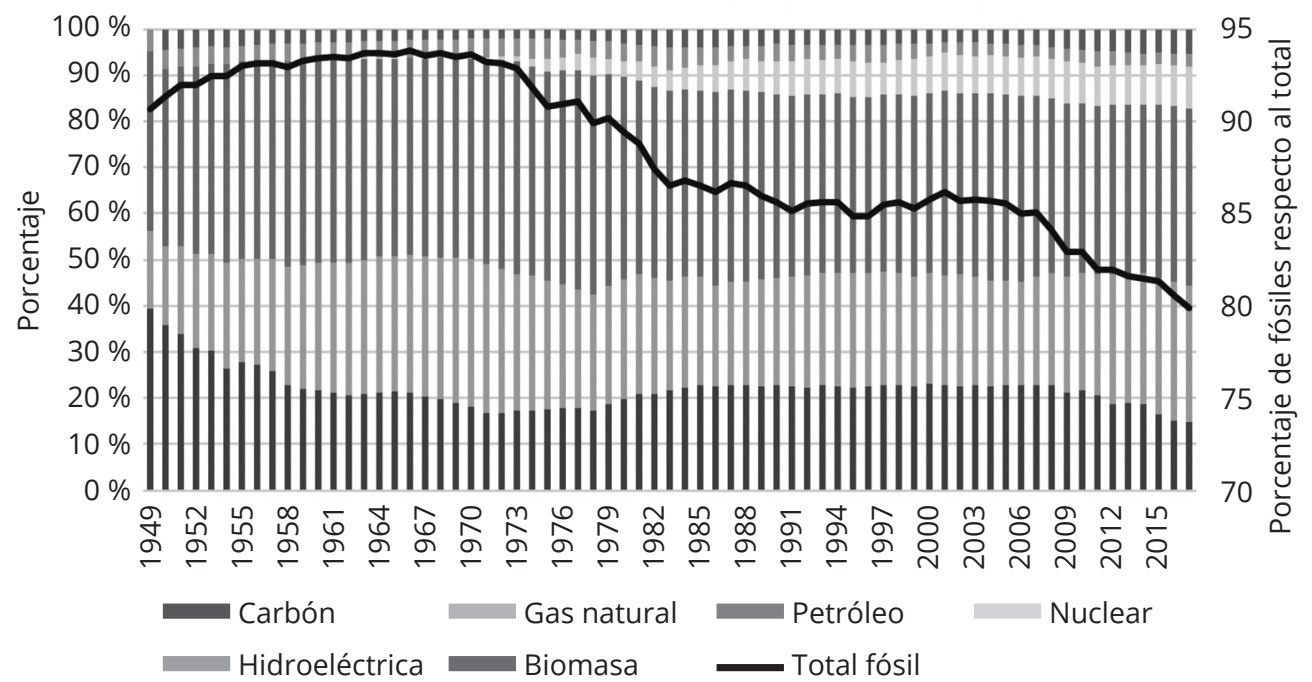

Fuente: elaboración propia con datos de la Energy Information Agency (2019).

Gráfica A 2. Distintos tipos de energía renovable, en porcentajes

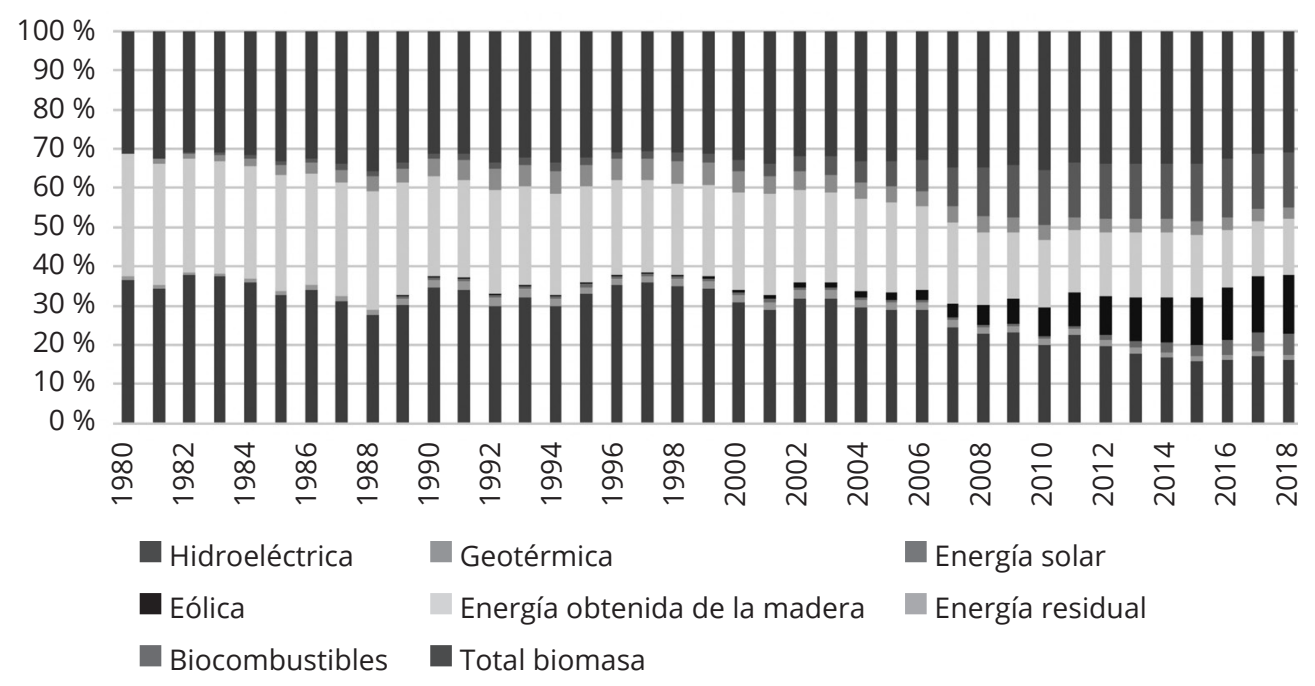

Fuente: elaboración propia con datos de la Energy Information Administration (2019). 


\section{Referencias bibliográficas}

Bacchetta, V. L. (2013). «Geopolítica del fracking: Impactos y riesgos ambientales». Nueva sociedad, (244): 61-73.

British Petroleum, BP (2019). Statistical Review of World Energy 2019 (68a ed.), https:// www.bp.com/content/dam/bp/business-sites/en/global/corporate/pdfs/energyeconomics/statistical-review/bp-stats-review-2019-full-report.pdf

Cheng, I.-H. y Xiong, W. (2013). «The Financialization of Commodity Markets». Presentado en la Annual Review of Financial Economics, octubre.

Cherp, A. y Jewell, J. (2014). «The concept of energy security: Beyond the four As». Energy Policy, 75: 415-421. https://doi.org/10.1016/j.enpol.2014.09.005

Enerdata (2019). Global Energy Statistical Yearbook. https://yearbook.enerdata.net/

Energy Information Administration (2019), Short Term Energy Outlook, ElA. https:// www.eia.gov/outlooks/steo/outlook.php

Federal Reserve Bank of St. Louis (2018). Inflation, consumer prices for the United States. Recuperado el 18 de diciembre de 2018 de: https://fred.stlouisfed.org/ series/FPCPITOTLZGUSA

Global Carbon Atlas (2020). Fossil fuels emissions. http://www.globalcarbonatlas.org/ en/CO2-emissions

Global Energy Institute (2019). Index of U.S. Energy Security Risk, Addressing America's Vulnerabilities in a Global Energy Market. EEUU: Global Energy Institute, Chamber of Commerce.

Hamilton, J. (2008). «Understanding crude oil prices», Working Paper 14492.

International Energy Agency, IEA (2011a). The IEA Model of Short-term Energy Security (MOSES) Primary Energy Sources and Secondary Fuels, IEA/ OECD.

International Energy Agency, IEA (2011b). «Energy security. Ensuring the uninterrupted availability of energy sources at an affordable price». IEA. Recuperado el 20 de febrero de 2020 de: https://www.iea.org/areas-of-work/ensuringenergy-security

International Energy Agency, IEA (2019). “Energy Security. Reliable, affordable access to all fuels and energy sources". IEA. Recuperado el 7 de mayo de en: https:// www.iea.org/topics/energysecurity/

Kim, B. (2015). «A New Method of Using Energy Security Risk as a Decision-Making Tool». Journal of Science Policy \& Governance. Vol. 6 (1). 
Kobek, M. L. P., Ugarte, A. y Aguilar, G. C. (2015). «Shale gas in the United States: transforming energy security in the twenty-first century». Norteamérica, 10 (1): 7-38. https://doi.org/10.20999/nam.2015.a001

Krane, J y Medlock, K. B. (2018). «Geopolitical dimensions of US oil security». Energy Policy, vol. 114: 558-565.

Naciones Unidas (2015). Objetivos de Desarrollo Sostenible. Recuperado el 20 de diciembre de 2019 de: https://www.un.org/sustainabledevelopment/es/

National Security Council (1979). Memorandum from Director of Central Intelligence Turner to the President's Assistant for National Security Affairs (Brzezinski). Recuperado el 2 de octubre de 2018 de http://history.state.gov/historicaldocuments/ frus1969-76v37/d240

Pandey, A. (2018). «Energy: A Basic Human Right», Energy Monitor. https://www. geopoliticalmonitor.com/energy-a-basic-human-right/

Puyana, A. (2015). La economía petrolera en un mercado politizado y global. México y Colombia. México: FLACSO.

Puyana A. y Rodríguez E. (2019). «Seguridad energética. ¿Un objetivo lograble? La trayectoria en México, Estados Unidos y Canadá de 1980 al 2016» (en dictaminación).

Sampson, A. (1975). The Seven Sisters: The Great Oil Companies and the World They Shaped. Nueva York: Bantam.

Sovacool, B. K. (2013). «An international assessment of energy security performance». Ecological Economics, 88: 148-158.

Sovacool, B. K. y Mukherjee, I. (2011). «Conceptualizing and measuring energy security: A synthesized approach». Energy, 36 (8): 5343-5355.

Winzer, C. (2012). «Conceptualizing energy security». Energy Policy, vol. 46: 36-48.

World Energy Council (2007). «Energy and Climate Change World». Reino Unido: Energy Council.

Yergin, D. (1991). The Prize: The Epic Quest for Oil, Money \& Power. Nueva York, Londres, Toronto: Free Press. 